\title{
Constrained consistency enforcement in AHP
}

\author{
Julio Benítez, Silvia Carpitella† Antonella Certa; Joaquín Izquierdo ${ }^{\S}$
}

\begin{abstract}
Decision-making in the presence of intangible elements must be based on a robust, but subtle, balance between expert know-how and judgment consistency when eliciting that know-how. This balance is frequently achieved as a trade-off reached after a feedback process softens the tension frequently found between one force steadily pulling towards (full) consistency, and another force driven by expert feeling and opinion. The linearization method, developed by the authors in the framework of the analytic hierarchy process, is a pull-towards-consistency mechanism that shows the path from an inconsistent body of judgment elicited from an expert towards consistency, by suggesting optimal changes to the expert opinions. However, experts may be reluctant to alter some of their issued opinions, and may wish to impose constraints on the adjustments suggested by the consistency-enforcement mechanism. In this paper, using the classical Riesz representation theorem, the linearization method is accommodated to consider various types of constraints imposed by experts during the abovementioned feedback process.
\end{abstract}

Keywords: Decision-making, expert judgment, consistency, consensus, AHP

\section{Introduction and literature review}

Making sound and effective decisions is crucial in real-world optimization processes involving difficult to quantify variables. In this case, decision-making (DM) is commonly driven by the personal experience of a single stakeholder, or often by the consensual involvement of a group of decision makers [1]. It is a fact that individuals and enterprises rely on multi-criteria decision-making (MCDM) methods to achieve wise and effective solutions [2] for many of their problems. The existing literature offers many examples $[3,4,5,6]$ that demonstrate the successful application - also under hybrid arrangements [7] - of MCDM methods to resolve a wide variety of multi-criteria optimization problems $[8,9,10,11]$.

However, far from being straightforward, DM is plagued with difficulties for a number of reasons.

After having briefly introduced the role of MCDM methods and their usage in the literature, Erdogan et al. [12] report the steps necessary to use these techniques. Starting from a clear definition of the sets of options (usually called alternatives) and criteria, a mechanism to evaluate those elements should be established to calculate (quantify) their relative and mutual importance in terms of weights. Such a mechanism should cope with various delicate features.

Subjectivity. Ability to make sensible use of the subjective perception of experts is crucial.

Uncertainty. Contexts characterized by uncertainty $[13,14]$ need techniques tailored to cope with it.

Incompleteness. Lack of information is also frequent in elucidation [15] and must be suitably handled.

\footnotetext{
*IMM, Universitat Politècnica de València, Valencia, Spain.

†Flulng-IMM, Universitat Politècnica de València, Valencia, Spain - DIID, Università degli Studi di Palermo, Palermo, Italy.

tDIID, Università degli Studi di Palermo, Palermo, Italy.

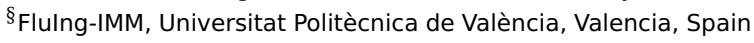


Consensus. When various decision makers participate in the evaluations, consensus within the group is central. Kozierkiewicz-Hetmańska [16] develops a set of new theorems focusing on the enhancement of consensus quality when dealing with collective knowledge.

Disagreement. Dror et al. [17] refers to the reliability of DM processes and recognizes disagreement among experts in a DM group as cause for concern. The authors underline the importance of collecting contextual information and assert that even the same expert, when examining the same data on different occasions, may reach different conclusions. In fact, as asserted by Rekha and Muccini [18], involving groups can increase both the level of complexity and the opportunities for DM methods. Montani et al. [19] develop a framework aimed at handling scenarios in which opinions given by two experts with similar backgrounds diverge, and attempts at reconciliation to obtain a final result.

Negotiation. Exchanging feedback with experts [20] to ensure that acceptable and consistent values are in line with the real perception of phenomena by experts is essential. Pérez et al. [21] discuss a consensus-reaching process as a negotiation problem, in which discussion and deliberation among the group members are facilitated for sharing opinions and agreeing on a final decision.

Hard constraints. During negotiation, however, cases may occur in which one or more experts do not wish to modify part of their judgments, or anchor specific constraints to be satisfied. To the best of our knowledge, the consideration of hard constraints during feedback processes is an aspect not yet considered from a mathematical perspective.

Integrating these kinds of aspects in MCDM models is paramount for DM actions to be structured and supported by reliable tools that can cope with the above issues. In particular, a structured tool would facilitate the finding of a good trade-off among diverse options in a consistent way, and should include expert hard-constraints. This is the main subject of this paper, and which we approach within the analytic hierarchy process (AHP) framework.

The AHP, first developed by Thomas Saaty [22], is one of the easiest and most flexible MCDM methods [23]. Based on pairwise comparisons between the elements under consideration, it enables synthetizing judgments, even if issued by multiple actors, to make a good assessment of the alternatives [24] under variously weighted criteria [25]. A crucial point within the AHP framework is the measurement of the expert consistency [26] when elucidating comparisons of pairs of elements. These comparisons are translated into numbers using a numerical scale [27], and then collected in a matrix.

In this way, a body of knowledge is compiled in a pairwise comparison matrix (PCM), whose entries express the numerical evaluations that embody expert opinions about the relative importance of elements [28]. As highlighted by [29], PCMs are a popular tool for weighting criteria and, consequently, evaluating alternatives in relation to a given DM process. However, experts may produce inconsistent judgments [30], especially when comparing a large number of elements [31], due to a natural lack of consistency in human thinking [32]. To help solve this problem, in a previous work [33], the authors developed a linearization process to calculate the closest (synthetic) fully consistent matrix to a given PCM. However, fully consistent values may (significantly) differ from expert evaluations, and from what is the real (and most practical) expert perspective. To bridge this gap between the mentioned linearization process outcome and reality, the importance of exchanging feedback with the experts was underlined [34]. The consequent trade-off process aims to achieve a final matrix with an "acceptable" level of consistency [35], while still reflecting expert personal experience as well as possible.

Regarding the present work, we were initially interested in exploring the case in which an expert rejects a change suggested by the consistency-enforcement method. In other terms, our first research question was: how can we achieve the closest consistent matrix to a PCM under the constraint that an expert wants to keep one of the original entries unchanged. This simple but important problem is here solved in a more general context, in which an expert or group of experts impose certain (hard) constraint(s) affecting the suggested modifications of their judgments, while not objecting 
to changing the remaining judgements. This generalization makes use of the classical Riesz representation theorem [36, Section 3.7]. The proposed approach simultaneously guarantees adherence to the expert opinions, and is consistent in dealing with evaluations when carrying out AHP decisions in the soundest manner.

This article is structured as follows. After this introduction and literature review, in Section 2 we present the necessary background. Section 3 motivates and clearly states the problem, and Section 4 (as the central section) develops the solution proposed through several technical lemmas and theorems, and presents several illustrative numerical examples. The codes used in the examples are given in the Appendix. Finally, the sections of conclusions and references close the paper.

\section{Background and used notation}

Two types of matrices arise in AHP theory. A reciprocal matrix $A=\left(a_{i j}\right)$ is a square $n \times n$ matrix that satisfies $a_{i j}>0$ and $a_{i j} a_{j i}=1$ for all $1 \leq i, j \leq n$. A consistent matrix $A=\left(a_{i j}\right)$ is a square $n \times n$ matrix that satisfies $a_{i j}>0$ and $a_{i j} a_{j k}=a_{i k}$ for all $1 \leq i, j, k \leq n$. It is trivial to prove that any consistent matrix is reciprocal. Also, it can be easily proved that the rank of any consistent matrix is 1 [37, Theorem 1].

The set of $n \times m$ real matrices will be denoted by $\mathscr{M}_{n, m}$. We write $\mathscr{M}_{n, m}^{+}=\left\{\left(a_{i j}\right) \in \mathscr{M}_{n, m}: a_{i j}>\right.$ 0 for all $i, j\}$. If $A$ is a matrix, then $\operatorname{tr}(A)$ and $A^{T}$ will denote the trace and the transpose of $A$, respectively. Any vector of $\mathbb{R}^{n}$ will be considered a column. We denote by $\mathbf{e}_{1}, \ldots, \mathbf{e}_{n}$ the vectors of the standard basis of $\mathbb{R}^{n}$. If $\mathbf{x}_{1}, \ldots, \mathbf{x}_{k} \in \mathbb{R}^{n}$, we denote by $\operatorname{span}\left\{\mathbf{x}_{1}, \ldots, \mathbf{x}_{k}\right\}$ the subspace spanned by these vectors. The vector $[1 \cdots 1]^{T} \in \mathbb{R}^{n}$ will be denoted by $\mathbb{1}_{n}$.

We will use the mappings $L: \mathscr{M}_{n, m}^{+} \rightarrow \mathscr{M}_{n, m}$ and $E: \mathscr{M}_{n, m} \rightarrow \mathscr{M}_{n, m}^{+}$given by $(L(A))_{i j}=\left(\log \left(a_{i j}\right)\right)$ and $(E(A))_{i j}=\left(\exp \left(a_{i j}\right)\right)$, respectively, where $A=\left(a_{i j}\right)$. Evidently, for $A \in \mathscr{M}_{n, m}^{+}$, one has that $A$ is reciprocal if and only if $L(A)$ is skew-symmetric.

For two positive matrices $A, B \in \mathscr{M}_{n, m}$, we define

$$
\mathrm{d}(A, B)=\|L(A)-L(B)\|_{F},
$$

where $\|\cdot\|_{F}$ is the Frobenius norm. It is well known that $\|A\|_{F}=\operatorname{tr}\left(A A^{T}\right)^{1 / 2}$ holds for any matrix $A$. Also, it is well known that the rule

$$
\langle A, B\rangle=\operatorname{tr}\left(A B^{T}\right)
$$

defines an inner product in $\mathscr{M}_{n, m}$.

For a reciprocal matrix $A \in \mathscr{M}_{n, n}$, it is proved in [33, Theorem 2.3] that there is a unique consistent matrix, denoted by $X_{A}$, such that

$$
\mathrm{d}\left(A, X_{A}\right)=\min \left\{\mathrm{d}(A, X): X \in \mathscr{M}_{n, n}, X \text { is consistent }\right\} .
$$

This matrix $X_{A}$ is obtained by means of the linearisation process:

$$
\begin{aligned}
& \text { Reciprocal matrices } \rightarrow \mathscr{S}_{n} \rightarrow \mathscr{L}_{n} \rightarrow \text { Consistent matrices } \\
& A \quad \mapsto L(A) \mapsto p_{n}(L(A)) \quad \mapsto \quad X_{A}=E\left(p_{n}(L(A))\right)
\end{aligned}
$$

Here, $\mathscr{S}_{n}$ is the set of $n \times n$ skew-symmetric matrices, $\mathscr{L}_{n}=\left\{L(M): M \in \mathscr{M}_{n, n}, M\right.$ is consistent $\}$, and $p_{n}: \mathscr{M}_{n, n} \rightarrow \mathscr{L}_{n}$ is the orthogonal projection assuming that $\mathscr{M}_{n, n}$ is endowed with the inner product defined in (2).

It can be proved (see [33, Theorem 2.2]) that $\mathscr{L}_{n}$ is a linear subspace with $\operatorname{dim} \mathscr{L}_{n}=n-1$. In fact, if we define the linear mapping $\phi_{n}: \mathbb{R}^{n} \rightarrow \mathscr{M}_{n, n}$ given by

$$
\phi_{n}(\mathbf{x})=\mathbf{x} \mathbb{1}_{n}^{T}-\mathbb{1}_{n} \mathbf{x}^{T}
$$

it is seen that $\operatorname{ker} \phi_{n}$ is the subspace spanned by $\mathbb{1}_{n}$, and $\operatorname{Im} \phi_{n}=\mathscr{L}_{n}$. 
Fact 1 If $\mathbf{v}=\left[\begin{array}{lll}v_{1} & \cdots & v_{n}\end{array}\right]^{T} \in \mathbb{R}^{n}$, then, by the previous considerations, $\phi_{n}(\mathbf{v}) \in \mathscr{L}_{n}$, and therefore, $E\left(\phi_{n}(\mathbf{v})\right)$ is consistent. But the $(i, j)$ entry of $E\left(\phi_{n}(\mathbf{v})\right)$ is $\exp \left(v_{i}-v_{j}\right)=\exp \left(v_{i}\right) / \exp \left(v_{j}\right)$. Thus, the vector $E(\mathbf{v})$ is a priority vector of $E\left(\phi_{n}(\mathbf{v})\right)$. Recall that if $\mathbf{w}$ is a priority vector of a consistent matrix, then $K \mathbf{w}$ is also a priority vector for any $K>0$.

The matrix obtained in the linearisation process (3) can be computed by means of the following expression (see [38, Theorem 3]) of $p_{n}(M)$ for an arbitrary $M \in \mathscr{S}_{n}$ :

$$
p_{n}(M)=\frac{1}{n}\left[\left(M U_{n}\right)-\left(M U_{n}\right)^{T}\right]
$$

where $U_{n}=\mathbb{1}_{n} \mathbb{1}_{n}^{T} \in \mathscr{M}_{n, n}$ is the matrix all of whose components are ones.

\section{Motivation and general setting of the problem}

To motivate and get a better understanding of the main results of the paper, we begin by setting a previous problem and providing an example.

Problem 1 Let $A \in \mathscr{M}_{n, n}$ be a reciprocal matrix. Find a consistent matrix which is the closest to $A$ such that the entries $(1,2)$ of $A$ and this consistent matrix are equal. It is considered the distance defined in (1).

Evidently, we can assume that $n>2$, since otherwise, the whole matrix $A$ would be fixed.

Example 2 Let $A$ be the following reciprocal matrix:

$$
A=\left[\begin{array}{ccc}
1 & 2 & 2 \\
1 / 2 & 1 & 3 \\
1 / 2 & 1 / 3 & 1
\end{array}\right]
$$

The consistent matrix closest to $A$ in the sense of the distance (1) can be computed by using (5):

$$
X_{A}=\left[\begin{array}{ccc}
1 & 1.387 & 2.885 \\
0.721 & 1 & 2.080 \\
0.347 & 0.481 & 1
\end{array}\right]
$$

It is clear that all the entries of $A$ have changed. It is feasible that the expert having produced matrix $A$ does not want to see a certain entry of $A$ altered. For example, if $\mathbf{x}=\left[\begin{array}{lll}x_{1} & x_{2} & x_{3}\end{array}\right]^{T} \in \mathbb{R}^{3}$ is the normalised priority vector, the expert wants that $x_{1}=2 x_{2}$. In this case, the expert wants that $a_{12}=x_{1} / x_{2}=2$ is unchangeable.

The previous Problem 1 can be generalised as follows:

Problem 2 Let $B=\left(b_{i j}\right) \in \mathscr{M}_{n, n}$ be a skew-symmetric matrix and $c \in \mathbb{R}$. Find $Y=\left(y_{i j}\right) \in \mathscr{L}_{n}$ such that

$$
\|B-Y\|_{F} \leq\left\|B-Y^{\prime}\right\|_{F} \quad \text { and } \quad y_{12}=c
$$

for all $Y^{\prime} \in \mathscr{L}_{n}$ such that the entry $(1,2)$ of $Y^{\prime}$ is $c$.

Observe that if $c=b_{12}$, then Problem 2 is exactly Problem 1 .

We will generalise Problem 2 in two ways. One way is evident: to increase the number of fixed entries of the skew-symmetric matrix $B$. The other direction will be explained in the next paragraph.

Observe that the mapping $\Psi: \mathscr{M}_{n, n} \rightarrow \mathbb{R}$ given by $\Psi(M)=m_{12}$ is linear, and fixing the entry $(1,2)$ in a matrix $M$ is equivalent to fix the value $\Psi(M)$. Therefore, another way to generalise Problem 2 is fixing $\Psi(M)$ for a given linear mapping $\Psi: \mathscr{M}_{n, n} \rightarrow \mathbb{R}$. Instead of working with linear mappings from $\mathscr{M}_{n, n}$ to $\mathbb{R}$ we will deal with more familiar objects, as we will show in next paragraph.

Let us note that if $\Psi: \mathscr{M}_{n, n} \rightarrow \mathbb{R}$ is a linear mapping, then there exists a unique matrix $R \in \mathscr{M}_{n, n}$ such that $\Psi(M)=\langle M, R\rangle$ for any $M \in \mathscr{M}_{n, n}$ (this is part of the well-known Riesz representation 
theorem, see e.g. [36, Section 3.7]). Of course, we assume that $\mathscr{M}_{n, n}$ is endowed with the inner product defined in (2). Therefore, we can avoid the use of linear mapping from $\mathscr{M}_{n, n}$ to $\mathbb{R}$. Let us see a simple but useful lemma that permits translating linear operators to matrices. In the proof of this lemma we will use the equality $\operatorname{tr}(A B)=\operatorname{tr}(B A)$ valid for any pair of matrices $A, B$ such that $A B$ and $B A$ are meaningful.

Lemma 1 If $R=\left(r_{i j}\right) \in \mathscr{M}_{n, n}$, then $\left\langle\mathbf{e}_{i} \mathbf{e}_{j}^{T}, R\right\rangle=r_{i j}$.

Proof: It follows from

$$
\left\langle\mathbf{e}_{i} \mathbf{e}_{j}^{T}, R\right\rangle=\operatorname{tr}\left(\left(\mathbf{e}_{i} \mathbf{e}_{j}^{T}\right)^{T} R\right)=\operatorname{tr}\left(\mathbf{e}_{j} \mathbf{e}_{i}^{T} R\right)=\operatorname{tr}\left(\mathbf{e}_{i}^{T} R \mathbf{e}_{j}\right)=r_{i j}
$$

This expression is useful to find the matrix $R$ such that $\Psi(M)=\langle M, R\rangle$ for any $M \in \mathscr{M}_{n, n}$, where $\Psi: \mathscr{M}_{n, n} \rightarrow \mathbb{R}$ is a linear mapping, as we show in the next example.

Example 3 Let $\Psi: \mathscr{M}_{3,3} \rightarrow \mathbb{R}$ given by $\Psi(M)=m_{12}+m_{23}-m_{13}$. We shall find the matrix $R$ such that $\Psi(M)=\langle M, R\rangle$ for any $M \in \mathscr{M}_{3,3}$. By using $\langle M, R\rangle=m_{12}+m_{23}-m_{13}$ for matrices $M=\mathbf{e}_{i} \mathbf{e}_{j}^{T}$ and Lemma 1 we get

$$
R=\left[\begin{array}{rrr}
0 & 1 & -1 \\
0 & 0 & 1 \\
0 & 0 & 0
\end{array}\right]
$$

Now, we extend Problem 2. To this end, we introduce the following notation: for $R_{1}, \ldots, R_{k} \in \mathscr{M}_{n, n}$ and $c_{1}, \ldots, c_{k} \in \mathbb{R}$, we define

$$
\mathscr{A}\left(R_{1}, \ldots, R_{k}, c_{1}, \ldots, c_{k}\right)=\left\{M \in \mathscr{L}_{n}:\left\langle M, R_{i}\right\rangle=c_{i}, i=1, \ldots, k\right\} .
$$

When there is no danger of confusion, we simply write $\mathscr{A}$ instead of $\mathscr{A}\left(R_{1}, \ldots, R_{k}, c_{1}, \ldots, c_{k}\right)$. Recall that the set $\mathscr{L}_{n}$ is composed of $n \times n$ matrices of the form $L(X)$, where $X \in \mathscr{M}_{n, n}$ is consistent. Since $\operatorname{Im} \phi_{n}=\mathscr{L}_{n}$, where the linear mapping $\phi_{n}: \mathbb{R}^{n} \rightarrow \mathscr{M}_{n, n}$ is defined in (4), the above set $\mathscr{A}$ can be rewritten as

$$
\mathscr{A}\left(R_{1}, \ldots, R_{k}, c_{1}, \ldots, c_{k}\right)=\left\{\phi_{n}(\mathbf{v}): \mathbf{v} \in \mathbb{R}^{n},\left\langle\phi_{n}(\mathbf{v}), R_{i}\right\rangle=c_{i}, i=1, \ldots, k\right\}
$$

The generalisation of Problem 2 is the following one:

Problem 3 Let $B \in \mathscr{M}_{n, n}$ be skew-symmetric, $R_{1}, \ldots, R_{k} \in \mathscr{M}_{n, n}$, and $c_{1}, \ldots, c_{k} \in \mathbb{R}$. Find $Y \in \mathscr{A}$ such that

$$
\|B-Y\|_{F} \leq\left\|B-Y^{\prime}\right\|_{F} \quad \text { for all } Y^{\prime} \in \mathscr{A} .
$$

Where the set $\mathscr{A}$ is defined in (6).

Let $B \in \mathscr{M}_{n, n}$ be a skew-symmetric matrix. Notice that if $R=\left(r_{i j}\right) \in \mathscr{M}_{n, n}$ is defined as $r_{12}=1$ and $r_{i j}=0$ for $(i, j) \neq(1,2)$, then Problem 3 for $\mathscr{A}(R, c)$ reduces to Problem 2 . Evidently, for this matrix $R$ we have $\langle M, R\rangle=m_{12}$ for any matrix $M=\left(m_{i j}\right) \in \mathscr{M}_{n, n}$.

\section{The solution to Problem 3}

First of all, observe that if the set $\mathscr{A}\left(R_{1}, \ldots, R_{k}, c_{1}, \ldots, c_{k}\right)$ is empty, Problem 3 is meaningless. For example, let us consider the matrix $R$ of Example 3 (or the linear mapping $\Psi: \mathscr{M}_{3,3} \rightarrow \mathbb{R}$ given by $\left.\Psi(M)=m_{12}+m_{23}-m_{13}\right)$. Evidently, for any $\mathbf{v}=\left[\begin{array}{lll}v_{1} & v_{2} & v_{3}\end{array}\right]^{T} \in \mathbb{R}^{3}$, one has $\Psi\left(\phi_{n}(\mathbf{v})\right)=\left(\phi_{n}(\mathbf{v})\right)_{12}+$ $\left(\phi_{n}(\mathbf{v})\right)_{23}-\left(\phi_{n}(\mathbf{v})\right)_{13}=\left(v_{1}-v_{2}\right)+\left(v_{2}-v_{3}\right)-\left(v_{1}-v_{3}\right)=0$. Therefore, for this example, the set $\mathscr{A}(R, c)$ is empty for any $c \neq 0$. In the next Theorem 2 we shall characterise this "anomalous" behaviour. First, we see a useful result. 
Theorem 1 Let $R \in \mathscr{M}_{n, n}$ and $\mathbf{v} \in \mathbb{R}^{n}$. Then $\left\langle\phi_{n}(\mathbf{v}), R\right\rangle=\mathbf{v}^{T}\left(R \mathbb{1}_{n}-R^{T} \mathbb{1}_{n}\right)$.

Proof: According to the definition of $\phi_{n}$ given in (4), one has

$$
\left\langle\phi_{n}(\mathbf{v}), R\right\rangle=\operatorname{tr}\left(\phi_{n}(\mathbf{v})^{T} R\right)=\operatorname{tr}\left(\left(\mathbb{1}_{n} \mathbf{v}^{T}-\mathbf{v} \mathbb{1}_{n}^{T}\right) R\right)=\operatorname{tr}\left(\mathbb{1}_{n} \mathbf{v}^{T} R\right)-\operatorname{tr}\left(\mathbf{v} \mathbb{1}_{n}^{T} R\right) .
$$

We use that $\operatorname{tr}(A B)=\operatorname{tr}(B A)$ for any pair of matrices such that $A B$ and $B A$ are meaningful, and also $\operatorname{tr}(C)=\operatorname{tr}\left(C^{T}\right)$ for any square matrix $C$.

$$
\left\langle\phi_{n}(\mathbf{v}), R\right\rangle=\operatorname{tr}\left(\mathbf{v}^{T} R \mathbb{1}_{n}\right)-\operatorname{tr}\left(\left(\mathbf{v} \mathbb{1}_{n}^{T} R\right)^{T}\right)=\operatorname{tr}\left(\mathbf{v}^{T} R \mathbb{1}_{n}\right)-\operatorname{tr}\left(R^{T} \mathbb{1}_{n} \mathbf{v}^{T}\right)=\operatorname{tr}\left(\mathbf{v}^{T} R \mathbb{1}_{n}\right)-\operatorname{tr}\left(\mathbf{v}^{T} R^{T} \mathbb{1}_{n}\right) .
$$

Having in mind that $\mathbf{v}^{T} R \mathbb{1}_{n}$ and $\mathbf{v}^{T} R^{T} \mathbb{1}_{n}$ are scalars, one obtains that

$$
\left\langle\phi_{n}(\mathbf{v}), R\right\rangle=\mathbf{v}^{T} R \mathbb{1}_{n}-\mathbf{v}^{T} R^{T} \mathbb{1}_{n}=\mathbf{v}^{T}\left(R-R^{T}\right) \mathbb{1}_{n} .
$$

Incidentally, Theorem 1 tells that the adjoint of $\phi_{n}: \mathbb{R}^{n} \rightarrow \mathscr{M}_{n, n}$ is given by $\phi_{n}^{*}: \mathscr{M}_{n, n} \rightarrow \mathbb{R}^{n}$, $\phi_{n}^{*}(R)=R \mathbb{1}_{n}-R^{T} \mathbb{1}_{n}$. This theorem has the following consequence.

Theorem 2 Let $R \in \mathscr{M}_{n, n}$. The following affirmations are equivalent.

a) $\left\langle R, \phi_{n}(\mathbf{v})\right\rangle=0$ for all $\mathbf{v} \in \mathbb{R}^{n}$.

b) $R \mathbb{1}_{n}=R^{T} \mathbb{1}_{n}$.

Example 4 Let $R$ be the matrix of Example 3. Since $R \mathbb{1}_{3}=R^{T} \mathbb{1}_{3}$, by Theorem 2, we have $\left\langle R, \phi_{3}(\mathbf{v})\right\rangle=$ 0 for all $\mathbf{v} \in \mathbb{R}^{3}$ (we already knew this fact from the previous paragraph to Theorem 1 ).

By Theorem 1 , the the set $\mathscr{A}\left(R_{1}, \ldots, R_{k}, c_{1}, \ldots, c_{k}\right)$ defined in (7) can be described in a simpler way:

$$
\mathscr{A}\left(R_{1}, \ldots, R_{k}, c_{1}, \ldots, c_{k}\right)=\left\{\phi_{n}(\mathbf{v}): \mathbf{v} \in \mathbb{R}^{n}, \mathbf{v}^{T}\left(R_{i} \mathbb{1}_{n}-R_{i}^{T} \mathbb{1}_{n}\right)=c_{i}, i=1, \ldots, k\right\} .
$$

A technical result is given in the next lemma.

Lemma 2 If $M \in \mathscr{M}_{n, n}$ is any matrix, then $\mathbb{1}_{n}^{T}\left(M-M^{T}\right) \mathbb{1}_{n}=0$.

Proof: Let $\theta=\mathbb{1}_{n}^{T}\left(M-M^{T}\right) \mathbb{1}_{n}$. Since $\theta$ is a scalar, we get

$$
\theta=\theta^{T}=\left(\mathbb{1}_{n}^{T}\left(M-M^{T}\right) \mathbb{1}_{n}\right)^{T}=\mathbb{1}_{n}^{T}\left(M^{T}-M\right) \mathbb{1}_{n}=-\theta .
$$

Therefore, $\theta=0$.

The following result explains the algebraic structure of $\mathscr{A}$ written in (8). If $G$ is any matrix, we will denote by $\mathscr{N}(G)$ and $\mathscr{R}(G)$ the null space and the range space, respectively, of $G$.

Theorem 3 Let $R_{1}, \ldots, R_{k} \in \mathscr{M}_{n, n}$ and $c_{1}, \ldots, c_{k} \in \mathbb{R}$. Let us define

$$
\mathbf{x}_{i}=\left(R_{i}-R_{i}^{T}\right) \mathbb{1}_{n}, i=1, \ldots, k, \quad G=\left[\mathbf{x}_{1} \cdots \mathbf{x}_{k}\right] .
$$

a) If $\left\{\mathbf{w} \in \mathbb{R}^{n}: \mathbf{w}^{T} \mathbf{x}_{i}=c_{i}, i=1, \ldots, n\right\}=\varnothing$, then $\mathscr{A}\left(R_{1}, \ldots, R_{k}, c_{1}, \ldots, c_{k}\right)=\varnothing$.

b) If there exists $\mathbf{w}_{0} \in \mathbb{R}^{n}$ such that $\mathbf{w}_{0}^{T} \mathbf{x}_{i}=c_{i}$ for $i=1, \ldots, k$, then $\mathscr{A}\left(R_{1}, \ldots, R_{k}, c_{1}, \ldots, c_{k}\right)$ is a linear manifold whose dimension is $n-1-\operatorname{rk}(G)$.

Proof: Part a) is trivial to prove in view of (8). We will prove b) in the next paragraph (we will write $\mathscr{A}$ instead of $\mathscr{A}\left(R_{1}, \ldots, R_{k}, c_{1}, \ldots, c_{k}\right)$ for the sake of simplicity).

Since $\left\{\mathbf{w} \in \mathbb{R}^{n}: \mathbf{w}^{T} \mathbf{x}_{i}=c_{i}, i=1, \ldots, k\right\}$ is a non empty linear manifold in $\mathbb{R}^{n}$ and $\phi_{n}$ is a linear mapping, it is clear that $\mathscr{A}$ is a linear manifold in view of (8). Also, if $\mathscr{X}=\left\{\mathbf{w} \in \mathbb{R}^{n}: \mathbf{w}^{T} \mathbf{x}_{i}=0, i=\right.$ 
$1, \ldots, k\}$, then (8) yields $\operatorname{dim} \mathscr{A}=\operatorname{dim}\left(\left.\operatorname{Im} \phi_{n}\right|_{\mathscr{X}}\right)$, where $\left.\phi_{n}\right|_{\mathscr{X}}: \mathscr{X} \rightarrow \mathscr{M}_{n, n}$ denotes the restriction of $\phi_{n}$ to $\mathscr{X}$. Therefore, $\operatorname{dim} \mathscr{X}=\operatorname{dim}\left(\left.\operatorname{ker} \phi_{n}\right|_{\mathscr{X}}\right)+\operatorname{dim} \mathscr{A}$. It is evident that $\mathscr{X}=\mathscr{N}\left(G^{T}\right)$; hence by recalling that $G^{T} \in \mathscr{M}_{k, n}$, one has $\operatorname{dim} \mathscr{X}=\operatorname{dim} \mathscr{N}\left(G^{T}\right)=n-\operatorname{rk}\left(G^{T}\right)=n-\operatorname{rk}(G)$. To finish the proof of this theorem, it is enough to prove dim $\left.\operatorname{ker} \phi_{n}\right|_{\mathscr{X}}=1$. In fact, evidently, $\left.\operatorname{ker} \phi_{n}\right|_{\mathscr{X}}=\operatorname{ker} \phi_{n} \cap \mathscr{X}=$ $\operatorname{span}\left\{\mathbb{1}_{n}\right\} \cap \mathscr{X}$, but Lemma 2 leads to $\mathbb{1}_{n}^{T} \mathbf{x}_{i}=\mathbb{1}_{n}^{T}\left(R_{i}-R_{i}^{T}\right) \mathbb{1}=0$ for $i=1, \ldots, k$, hence $\mathbb{1}_{n} \in \mathscr{X}$, and thus, $\left.\operatorname{ker} \phi_{n}\right|_{\mathscr{X}}=\operatorname{span}\left\{\mathbb{1}_{n}\right\}$.

Remark 1 A key condition in Theorem 3 is the existence of $\mathbf{w}_{0} \in \mathbb{R}^{n}$ such that

$$
\mathbf{w}_{0}^{T} \mathbf{x}_{i}=c_{i} \quad \forall i=1, \ldots, k .
$$

If we define $\mathbf{c}=\left[\begin{array}{lll}c_{1} & \cdots & c_{k}\end{array}\right]^{T}$, then (10) is equivalent to $G^{T} \mathbf{w}_{0}=\mathbf{c}$, where the matrix $G$ has been defined in (9). Hence, the existence of such $\mathbf{w}_{0}$ is equivalent to the consistency of the linear system $G^{T} \mathbf{w}=\mathbf{c}$. By using the Moore-Penrose of $G^{T}$, denoted by $\left(G^{T}\right)^{\dagger}$, the following useful characterization may be obtained:

$$
\text { The system } G^{T} \mathbf{w}=\mathbf{c} \text { is consistent } \Leftrightarrow G^{T}\left(G^{T}\right)^{\dagger} \mathbf{c}=\mathbf{c} .
$$

In addition, if the system $G^{T} \mathbf{w}=\mathbf{c}$ is consistent, then $\left(G^{T}\right)^{\dagger} \mathbf{c}$ is a particular solution.

Let us observe that the proof of Thorem 3 distils $\operatorname{dim}\left(\phi_{n}\left(\mathscr{N}\left(G^{T}\right)\right)\right)=n-1-\operatorname{rk}(G)$. The next result gives the (theoretical) solution to Problem 3.

Theorem 4 Let $B \in \mathscr{M}_{n, n}$ be skew-symmetric, $R_{1}, \ldots, R_{k} \in \mathscr{M}_{n, n}$ and $c_{1}, \ldots, c_{k} \in \mathbb{R}$. Let us define $\mathbf{x}_{1}, \ldots, \mathbf{x}_{k}$ and $G$ as in (9). If there exists $\mathbf{w}_{0} \in \mathbb{R}^{n}$ such that $\mathbf{w}_{0}^{T} \mathbf{x}_{i}=c_{i}$ for $i=1, \ldots, k$, then the solution to Problem 3 is $Y=\phi_{n}\left(\mathbf{w}_{0}\right)+Z$, where $Z$ is the orthogonal projection onto $\phi_{n}\left(\mathscr{N}\left(G^{T}\right)\right)$ of $B-\phi_{n}\left(\mathbf{w}_{0}\right)$.

Proof: By (8), it is clear that $\mathscr{A}\left(R_{1}, \ldots, R_{k}, c_{1}, \ldots, c_{k}\right)=\phi_{n}\left(\mathbf{w}_{0}\right)+\phi_{n}\left(\mathscr{N}\left(G^{T}\right)\right)$. Therefore, find the minimum of $\|B-Y\|_{F}$ for $Y \in \mathscr{A}$ is equivalent to find the minimum of $\left\|B-\left(\phi_{n}\left(\mathbf{w}_{0}\right)+Z\right)\right\|_{F}$ for $Z \in \phi_{n}\left(\mathscr{N}\left(G^{T}\right)\right)$. The conclusion of the theorem is clear.

Since we must project (orthogonally) onto $\phi_{n}\left(\mathscr{N}\left(G^{T}\right)\right.$ ), we will find a basis for this subspace.

Theorem 5 Let $R_{1}, \ldots, R_{k} \in \mathscr{M}_{n, n}$ and $c_{1}, \ldots, c_{k} \in \mathbb{R}$. Let us define $\mathbf{x}_{i}$ and $G$ as in (8). Assume that there exists $\mathbf{w}_{0} \in \mathbb{R}^{n}$ such that $\mathbf{w}_{0}^{T} \mathbf{x}_{i}=c_{i}$ for $i=1, \ldots, k$. Let $r$ denote the rank of $G$.

a) There exists $\mathbf{u} \in \mathscr{N}\left(G^{T}\right)$ such that $\mathbf{u}^{T} \mathbb{1}_{n} \neq 0$.

b) If $\left\{\mathbf{u}_{1}, \ldots, \mathbf{u}_{n-r-1}, \mathbf{u}\right\}$ is an orthogonal basis of $\mathscr{N}\left(G^{T}\right)$, then $\left\{\phi_{n}\left(\mathbf{u}_{1}\right), \ldots, \phi_{n}\left(\mathbf{u}_{n-r-1}\right)\right\}$ is a basis of $\phi_{n}\left(\mathscr{N}\left(G^{T}\right)\right)$.

Proof: a) If $\mathscr{N}\left(G^{T}\right) \subset\left(\operatorname{span}\left\{\mathbb{1}_{n}\right\}\right)^{\perp}$, then $\operatorname{span}\left\{\mathbb{1}_{n}\right\} \subset \mathscr{N}\left(G^{T}\right)^{\perp}=\mathscr{R}(G)$. By the definition of matrix $G$ and vectors $\mathbf{x}_{i}$, we have that there exist $\lambda_{1}, \ldots, \lambda_{k} \in \mathbb{R}$ such that $\mathbb{1}_{n}=\sum_{i=1}^{k} \lambda_{i}\left(R_{i}-R_{i}^{T}\right) \mathbb{1}_{n}$. By Lemma 2, we get $n=\mathbb{1}_{n}^{T} \mathbb{1}_{n}=\sum_{i=1}^{k} \lambda_{i} \mathbb{1}_{n}^{T}\left(R_{i}-R_{i}^{T}\right) \mathbb{1}_{n}=0$, which is a contradiction. Hence a) follows.

b) Let $\left\{\mathbf{u}_{1}, \ldots, \mathbf{u}_{n-r-1}, \mathbf{u}\right\}$ be an orthogonal basis of $\mathscr{N}\left(G^{T}\right)$ (recall that $r$ is the rank of $G$ ). We will prove that $\left\{\phi_{n}\left(\mathbf{u}_{1}\right), \ldots, \phi_{n}\left(\mathbf{u}_{n-r-1}\right)\right\}$ is a basis of $\phi_{n}\left(\mathscr{N}\left(G^{T}\right)\right)$. By Theorem 3 , it is enough to prove that the vectors $\left\{\phi_{n}\left(\mathbf{u}_{1}\right), \ldots, \phi_{n}\left(\mathbf{u}_{n-r-1}\right)\right\}$ are linearly independent. To this end, let us consider $\sum_{s=1}^{n-r-1} \lambda_{s} \phi_{n}\left(\mathbf{u}_{s}\right)=\mathbf{0}$, where $\lambda_{1}, \ldots, \lambda_{n-r-1} \in \mathbb{R}$. It follows that $\sum_{s=1}^{n-r-1} \lambda_{s} \mathbf{u}_{s} \in \operatorname{ker} \phi_{n}=\operatorname{span}\left\{\mathbb{1}_{n}\right\}$. Therefore, there exists $\beta \in \mathbb{R}$ such that $\sum_{s=1}^{n-r-1} \lambda_{s} \mathbf{u}_{s}=\beta \mathbb{1}_{n}$. Since $\mathbf{u}^{T} \mathbf{u}_{s}=0$ for $s=1, \ldots, n-r-1$ (because $\left\{\mathbf{u}_{1}, \ldots, \mathbf{u}_{n-r-1}, \mathbf{u}\right\}$ is an orthogonal system), we get $\beta \mathbf{u}^{T} \mathbb{1}_{n}=0$, hence $\beta=0$ by item a), which leads to $\sum_{s=1}^{n-r-1} \lambda_{s} \mathbf{u}_{s}=\mathbf{0}$. We conclude that $\lambda_{1}=\cdots=\lambda_{n-r-1}=0$.

Remark 2 Octave provides the command null to find an orthonormal basis of the null space of any matrix by means of the singular value decomposition of this matrix (one can write type null in the Octave prompt to see the code). However, it cannot be assured that if null (G') is executed (to find the null space of $\mathcal{N}\left(G^{T}\right)$ ), the last vector of this subspace, $\mathbf{u}$, satisfies $\mathbf{u}^{T} \mathbb{1}_{n} \neq 0$.

Now comes another technical lemma useful in next Theorem 6. 
Lemma 3 Let $\mathbf{v}, \mathbf{w} \in \mathbb{R}^{n}$. Then $\left\langle\phi_{n}(\mathbf{v}), \phi_{n}(\mathbf{w})\right\rangle=2 n \mathbf{v}^{T} \mathbf{w}-2\left(\mathbf{v}^{T} \mathbb{1}_{n}\right)\left(\mathbf{w}^{T} \mathbb{1}_{n}\right)$.

Proof: We will use Theorem 1 and Definition 4.

$$
\begin{aligned}
\left\langle\phi_{n}(\mathbf{v}), \phi_{n}(\mathbf{w})\right\rangle & =\mathbf{v}^{T}\left[\phi_{n}(\mathbf{w})-\phi_{n}(\mathbf{w})^{T}\right] \mathbb{1}_{n} \\
& =\mathbf{v}^{T}\left[\left(\mathbf{w} \mathbb{1}_{n}^{T}-\mathbb{1}_{n} \mathbf{w}^{T}\right)-\left(\mathbf{w} \mathbb{1}_{n}^{T}-\mathbb{1}_{n} \mathbf{w}^{T}\right)^{T}\right] \mathbb{1}_{n} \\
& =2 \mathbf{v}^{T}\left(\mathbf{w} \mathbb{1}_{n}^{T}-\mathbb{1}_{n} \mathbf{w}^{T}\right) \mathbb{1}_{n} \\
& =2\left(\mathbf{v}^{T} \mathbf{w}\right)\left(\mathbb{1}_{n}^{T} \mathbb{1}_{n}\right)-2\left(\mathbf{v}^{T} \mathbb{1}_{n}\right)\left(\mathbf{w}^{T} \mathbb{1}_{n}\right) \\
& =2 n \mathbf{v}^{T} \mathbf{w}-2\left(\mathbf{v}^{T} \mathbb{1}_{n}\right)\left(\mathbf{w}^{T} \mathbb{1}_{n}\right) .
\end{aligned}
$$

The proof is finished.

Now we give an explicit solution to Problem 3. As is customary, $X^{\dagger}$ denotes the Moore-Penrose inverse of the matrix $X$.

Theorem 6 Let $B \in \mathscr{M}_{n, n}$ be skew-symmetric, $R_{1}, \ldots, R_{k} \in \mathscr{M}_{n, n}$ and $c_{1}, \ldots, c_{k} \in \mathbb{R}$. Let us define $\mathbf{x}_{i}$, $G$ as in (9), and $r=\operatorname{rk}(G)$. Assume that there exists $\mathbf{w}_{0} \in \mathbb{R}^{n}$ such that $\mathbf{w}_{0}^{T} \mathbf{x}_{i}=c_{i}$ for $i=1, \ldots, k$.

a) The solution to Problem 3 is $Y=\phi_{n}\left(\mathbf{w}_{0}+H \mu\right)$, where

(i) $\left\{\mathbf{u}_{1}, \ldots, \mathbf{u}_{n-r-1}, \mathbf{u}\right\}$ is an orthonormal basis of $\mathscr{N}\left(G^{T}\right), \mathbf{u}^{T} \mathbb{1}_{n} \neq 0$, and $H=\left[\mathbf{u}_{1} \cdots \mathbf{u}_{n-r-1}\right]$.

(ii) The vector $\mu \in \mathbb{R}^{n-r-1}$ is given by

$$
\boldsymbol{\mu}=\frac{1}{n} \boldsymbol{\beta}+\frac{1}{n\left(n-\left\|H^{T} \mathbb{1}_{n}\right\|^{2}\right)} H^{T} \mathbb{1}_{n} \mathbb{1}_{n}^{T} H \boldsymbol{\beta}, \quad \boldsymbol{\beta}=H^{T}\left[B \mathbb{1}_{n}-n \mathbf{w}_{0}+\left(\mathbf{w}_{0}^{T} \mathbb{1}_{n}\right) \mathbb{1}_{n}\right] .
$$

b) A priority vector of $E(Y)$ is $E\left(\mathbf{w}_{0}+H \boldsymbol{\mu}\right)$.

c) If we define $\mathbf{c}=\left[\begin{array}{llll}c_{1} & c_{2} & \cdots & c_{k}\end{array}\right]^{T}$, the vector $\mathbf{w}_{0}=\left(G^{T}\right)^{\dagger} \mathbf{c}$ satisfies $\mathbf{w}_{0}^{T} \mathbf{x}_{i}=c_{i}$ for $i=1, \ldots, k$ and the expression of $\boldsymbol{\beta}$ appearing in (12) reduces to $\boldsymbol{\beta}=H^{T} B \mathbb{1}_{n}$.

Proof: We apply Theorem 4 to get that the solution to Problem 3 is given by $Y=\phi_{n}\left(\mathbf{w}_{0}\right)+Z$, where $Z$ is the orthogonal projection onto $\phi_{n}\left(\mathscr{N}\left(G^{T}\right)\right)$ of $B-\phi_{n}\left(\mathbf{w}_{0}\right)$. By Theorem 5 , we can write $Z=$ $\sum_{s=1}^{n-r-1} \mu_{s} \phi_{n}\left(\mathbf{u}_{s}\right)$ for some real numbers $\mu_{s}$. Since $Z$ is the orthogonal projection onto $\phi_{n}\left(\mathscr{N}\left(G^{T}\right)\right)$ of $B-\phi_{n}\left(\mathbf{w}_{0}\right)$ and $\left\{\phi_{n}\left(\mathbf{u}_{1}\right), \ldots, \phi_{n}\left(\mathbf{u}_{n-r-1}\right)\right\}$ is a basis of $\phi_{n}\left(\mathscr{N}\left(G^{T}\right)\right)$, we obtain

$$
\left\langle Z-\left(B-\phi_{n}\left(\mathbf{w}_{0}\right)\right), \phi_{n}\left(\mathbf{u}_{i}\right)\right\rangle=0 \quad i=1, \ldots, n-r-1 .
$$

We shall simplify $\left\langle Z, \phi_{n}\left(\mathbf{u}_{1}\right)\right\rangle$ by using Lemma 3 and the orthonormality of $\left\{\mathbf{u}, \mathbf{u}_{1}, \ldots, \mathbf{u}_{n-r-1}\right\}$.

$$
\begin{aligned}
\left\langle Z, \phi_{n}\left(\mathbf{u}_{1}\right)\right\rangle & =\sum_{i=1}^{n-r-1} \mu_{i}\left\langle\phi_{n}\left(\mathbf{u}_{i}\right), \phi_{n}\left(\mathbf{u}_{1}\right)\right\rangle=\mu_{1}\left\langle\phi_{n}\left(\mathbf{u}_{1}\right), \phi_{n}\left(\mathbf{u}_{1}\right)\right\rangle+\sum_{i=2}^{n-r-1} \mu_{i}\left\langle\phi_{n}\left(\mathbf{u}_{i}\right), \phi_{n}\left(\mathbf{u}_{1}\right)\right\rangle \\
& =\left(2 n-2\left(\mathbf{u}_{1}^{T} \mathbb{1}_{n}\right)^{2}\right) \mu_{1}-2 \sum_{i=2}^{n-r-1}\left(\mathbf{u}_{i}^{T} \mathbb{1}_{n}\right)\left(\mathbf{u}_{1}^{T} \mathbb{1}_{n}\right) \mu_{i} .
\end{aligned}
$$

Analogous computations can be done for $\left\langle Z, \phi_{n}\left(\mathbf{u}_{i}\right)\right\rangle, i=2, \ldots, n-r-1$. We define

$$
\gamma_{i}=\left\langle B-\phi_{n}\left(\mathbf{w}_{0}\right), \phi_{n}\left(\mathbf{u}_{i}\right)\right\rangle=\left\langle B, \phi_{n}\left(\mathbf{u}_{i}\right)\right\rangle-\left(2 n \mathbf{w}_{0}^{T} \mathbf{u}_{i}-2\left(\mathbf{w}_{0}^{T} \mathbb{1}_{n}\right)\left(\mathbf{u}_{i}^{T} \mathbb{1}_{n}\right)\right)
$$

for $i=1, \ldots, n-r-1$. We obtain the linear system $C \mu=\gamma$, where

$$
C=\left[\begin{array}{cccc}
2 n-2\left(\mathbf{u}_{1}^{T} \mathbb{1}_{n}\right)^{2} & -2\left(\mathbf{u}_{1}^{T} \mathbb{1}_{n}\right)\left(\mathbf{u}_{2}^{T} \mathbb{1}_{n}\right) & \cdots & -2\left(\mathbf{u}_{1}^{T} \mathbb{1}_{n}\right)\left(\mathbf{u}_{n-r-1}^{T} \mathbb{1}_{n}\right) \\
-2\left(\mathbf{u}_{2}^{T} \mathbb{1}_{n}\right)\left(\mathbf{u}_{1}^{T} \mathbb{1}_{n}\right) & 2 n-2\left(\mathbf{u}_{2}^{T} \mathbb{1}_{n}\right)^{2} & \cdots & -2\left(\mathbf{u}_{2}^{T} \mathbb{1}_{n}\right)\left(\mathbf{u}_{n-r-1}^{T} \mathbb{1}_{n}\right) \\
\vdots & \vdots & \ddots & \vdots \\
-2\left(\mathbf{u}_{n-r-1}^{T} \mathbb{1}_{n}\right)\left(\mathbf{u}_{1}^{T} \mathbb{1}_{n}\right) & -2\left(\mathbf{u}_{n-r-1}^{T} \mathbb{1}_{n}\right)\left(\mathbf{u}_{2}^{T} \mathbb{1}_{n}\right) & \cdots & 2 n-2\left(\mathbf{u}_{n-r-1}^{T} \mathbb{1}_{n}\right)^{2}
\end{array}\right]
$$


$\boldsymbol{\mu}=\left[\mu_{1} \mu_{2} \cdots \mu_{n-r-1}\right]^{T}$, and $\boldsymbol{\gamma}=\left[\begin{array}{llll}\gamma_{1} & \gamma_{2} & \cdots & \gamma_{n-r-1}\end{array}\right]^{T}$.

Since $B$ is skew-symmetric, by Theorem 1 , one has $\left\langle B, \phi_{n}\left(\mathbf{u}_{i}\right)\right\rangle=\mathbf{u}_{i}^{T}\left(B-B^{T}\right) \mathbb{1}_{n}=2 \mathbf{u}_{i}^{T} B \mathbb{1}_{n}$. Therefore,

$$
\gamma_{i}=2\left(\mathbf{u}_{i}^{T} B \mathbb{1}_{n}-n \mathbf{u}_{i}^{T} \mathbf{w}_{0}+\left(\mathbf{w}_{0}^{T} \mathbb{1}_{n}\right)\left(\mathbf{u}_{i}^{T} \mathbb{1}_{n}\right)\right), \quad i=1, \ldots, n-r-1 .
$$

We can get a condensed way to write the vector $\gamma$ if we define $H=\left[\begin{array}{lll}\mathbf{u}_{1} & \cdots & \mathbf{u}_{n-r-1}\end{array}\right] \in \mathscr{M}_{n, n-r-1}$ :

$$
\gamma=2\left[H^{T} B \mathbb{1}_{n}-n H^{T} \mathbf{w}_{0}+\left(\mathbf{w}_{0}^{T} \mathbb{1}_{n}\right) H^{T} \mathbb{1}_{n}\right] .
$$

Set $\boldsymbol{\beta}=\boldsymbol{\gamma} / 2$ and let $M$ be the matrix such that $C=2 n I_{n-r-1}-2 M$. The system $C \boldsymbol{\mu}=\boldsymbol{\gamma}$ is equivalent to the system $\left(n I_{n-r-1}-M\right) \boldsymbol{\mu}=\boldsymbol{\beta}$. In the next paragraphs we will prove that the matrix $n I_{n-r-1}-M$ is non-singular and we will find an explicit expresion of $\left(n I_{n-r-1}-M\right)^{-1}$.

Observe that by using $\mathbf{u}_{i}^{T} \mathbb{1}_{n}=\mathbb{1}_{n}^{T} \mathbf{u}_{i}$ we have

$$
\begin{aligned}
M & =\left[\begin{array}{ccc}
\left(\mathbf{u}_{1}^{T} \mathbb{1}_{n}\right)^{2} & \cdots & \left(\mathbf{u}_{1}^{T} \mathbb{1}_{n}\right)\left(\mathbf{u}_{n-r-1}^{T} \mathbb{1}_{n}\right) \\
\vdots & \ddots & \vdots \\
\left(\mathbf{u}_{n-r-1}^{T} \mathbb{1}_{n}\right)\left(\mathbf{u}_{1}^{T} \mathbb{1}_{n}\right) & \cdots & \left(\mathbf{u}_{n-r-1}^{T} \mathbb{1}_{n}\right)^{2}
\end{array}\right]=\left[\begin{array}{c}
\mathbf{u}_{1}^{T} \mathbb{1}_{n} \\
\vdots \\
\mathbf{u}_{n-r-1}^{T} \mathbb{1}_{n}
\end{array}\right]\left[\begin{array}{lll}
\mathbf{u}_{1}^{T} \mathbb{1}_{n} & \cdots & \mathbf{u}_{n-r-1}^{T} \mathbb{1}_{n}
\end{array}\right] \\
& =\left[\begin{array}{c}
\mathbf{u}_{1}^{T} \\
\vdots \\
\mathbf{u}_{n-r-1}^{T}
\end{array}\right] \mathbb{1}_{n} \mathbb{1}_{n}^{T}\left[\begin{array}{lll}
\mathbf{u}_{1} & \cdots & \mathbf{u}_{n-r-1}
\end{array}\right]=H^{T} \mathbb{1}_{n} \mathbb{1}_{n}^{T} H .
\end{aligned}
$$

If we define $\alpha=\mathbb{1}_{n}^{T} H H^{T} \mathbb{1}_{n}=\left\|H^{T} \mathbb{1}_{n}\right\|^{2}$, then $M^{2}=H^{T} \mathbb{1}_{n} \mathbb{1}_{n}^{T} H H^{T} \mathbb{1}_{n} \mathbb{1}_{n}^{T} H=H^{T} \mathbb{1}_{n} \alpha \mathbb{1}_{n}^{T} H=\alpha M$.

In this paragraph we will prove that $\alpha \neq n$. If $\alpha=n$, then, by the Cauchy-Schwarz inequality, $n=\mathbb{1}_{n}^{T} H H^{T} \mathbb{1}_{n}=\mathbb{1}_{n}^{T}\left(H H^{T} \mathbb{1}_{n}\right) \leq\left\|\mathbb{1}_{n}\right\|\left\|H H^{T} \mathbb{1}_{n}\right\|$. But, since the system $\left\{\mathbf{u}_{1}, \ldots, \mathbf{u}_{n-r-1}\right\}$ is orthonormal, it is easy to see that the matrix $H H^{T}$ is the matrix of the orthogonal projection onto $\operatorname{span}\left\{\mathbf{u}_{1}, \ldots, \mathbf{u}_{n-r-1}\right\}$, hence $\left\|H H^{T} \mathbb{1}_{n}\right\| \leq\left\|\mathbb{1}_{n}\right\|$, and so $n \leq\left\|\mathbb{1}_{n}\right\|\left\|H H^{T} \mathbb{1}_{n}\right\| \leq\left\|\mathbb{1}_{n}\right\|^{2}=n$. Since the Cauchy-Scwharz inequality becomes an equality, the vectors $\mathbb{1}_{n}$ and $H H^{T} \mathbb{1}_{n}$ are linearly dependent, and so, there exists $\xi \in \mathbb{R}$ such that $H H^{T} \mathbb{1}_{n}=\xi \mathbb{1}_{n}$. By inserting this last equality into $n=\mathbb{1}_{n}^{T} H H^{T} \mathbb{1}_{n}$ we have $n=\mathbb{1}_{n}^{T}(\xi \mathbb{1})=\xi \mathbb{1}_{n}^{T} \mathbb{1}=\xi n$, and so, $\xi=1$, thus, $\mathbb{1}_{n}=H H^{T} \mathbb{1}_{n}$, in other words, $\mathbb{1}_{n}$ is equal to its orthogonal projection onto $\operatorname{span}\left\{\mathbf{u}_{1}, \ldots, \mathbf{u}_{n-r-1}\right\}$, and therefore, $\mathbb{1}_{n} \in \operatorname{span}\left\{\mathbf{u}_{1}, \ldots, \mathbf{u}_{n-r-1}\right\}$. By having in mind the orthogonality of the system $\left\{\mathbf{u}, \mathbf{u}_{1}, \ldots, \mathbf{u}_{n-r-1}\right\}$, we get $\mathbf{u}^{T} \mathbb{1}_{n}=0$, which is avoided by the hypotheses.

Now,

$$
\left(n I_{n-r-1}-M\right)\left[(n-\alpha) I_{n-r-1}+M\right]=n(n-\alpha) I_{n-r-1}+n M-(n-\alpha) M-M^{2}=n(n-\alpha) I_{n-r-1},
$$

which proves

$$
\left(n I_{n-r-1}-M\right)^{-1}=\frac{1}{n(n-\alpha)}\left[(n-\alpha) I_{n-r-1}+M\right] .
$$

Hence, $\left(n I_{n-r-1}-M\right) \boldsymbol{\mu}=\boldsymbol{\beta}$ leads to $\boldsymbol{\mu}=\left(n I_{n-r-1}-M\right)^{-1} \boldsymbol{\beta}$. Therefore, by recalling that $M=H^{T} \mathbb{1}_{n} \mathbb{1}_{n}^{T} H$ and $\alpha=\left\|H^{T} \mathbb{1}_{n}\right\|^{2}$, the vector $\mu$ can be written as in (12). Now, observe that the solution $Y^{n}$ to Problem 3 is

$$
Y=\phi_{n}\left(\mathbf{w}_{0}\right)+Z=\phi_{n}\left(\mathbf{w}_{0}\right)+\sum_{s=1}^{n-r-1} \mu_{s} \phi_{n}\left(\mathbf{u}_{s}\right)=\phi_{n}\left(\mathbf{w}_{0}+\sum_{s=1}^{n-r-1} \mu_{s} \mathbf{u}_{s}\right)=\phi_{n}\left(\mathbf{w}_{0}+H \boldsymbol{\mu}\right) .
$$

Item b) follows from Fact 1.

Let us prove c). Let $\mathbf{w}^{*}$ be any vector satisfying $\mathbf{x}_{i}^{T} \mathbf{w}^{*}=c_{i}$ for $i=1, \ldots, k$. Since $G^{T} \mathbf{w}^{*}=\mathbf{c}$, we have $G^{T}\left[\left(G^{T}\right)^{\dagger} \mathbf{c}\right]=G^{T}\left(G^{T}\right)^{\dagger} G^{T} \mathbf{w}^{*}=G^{T} \mathbf{w}^{*}=\mathbf{c}$. Therefore, we can choose $\mathbf{w}_{0}=\left(G^{T}\right)^{\dagger} \mathbf{c}$. With this choice, $\mathbf{w}_{0}^{T} \mathbb{1}_{n}=\left(\left(G^{T}\right)^{\dagger} \mathbf{c}\right)^{T} \mathbb{1}_{n}=\left(\left(G^{\dagger}\right)^{T} \mathbf{c}\right)^{T} \mathbb{1}_{n}=\mathbf{c}^{T} G^{\dagger} \mathbb{1}_{n}$. By using Lemma 2, we get

$$
G^{T} \mathbb{1}_{n}=\left[\begin{array}{c}
\mathbf{x}_{1}^{T} \\
\vdots \\
\mathbf{x}_{k}^{T}
\end{array}\right] \mathbb{1}_{n}=\left[\begin{array}{c}
\mathbf{x}_{1}^{T} \mathbb{1}_{n} \\
\vdots \\
\mathbf{x}_{k}^{T} \mathbb{1}_{n}
\end{array}\right]=\left[\begin{array}{c}
\mathbb{1}_{n}^{T} \mathbf{x}_{1} \\
\vdots \\
\mathbb{1}_{n}^{T} \mathbf{x}_{k}
\end{array}\right]=\left[\begin{array}{c}
\mathbb{1}_{n}^{T}\left(R_{1}-R_{1}^{T}\right) \mathbb{1}_{n} \\
\vdots \\
\mathbb{1}_{n}^{T}\left(R_{k}-R_{k}^{T}\right) \mathbb{1}_{n}
\end{array}\right]=\mathbf{0} .
$$


By recalling that $\mathscr{N}\left(G^{T}\right)=\mathscr{N}\left(G^{\dagger}\right)$, we have that $G^{\dagger} \mathbb{1}_{n}=\mathbf{0}$, hence $\mathbf{w}_{0}^{T} \mathbb{1}_{n}=0$.

Since $\mathbf{u}_{i} \in \mathscr{N}\left(G^{T}\right)=\mathscr{N}\left(G^{\dagger}\right)$ for $i=1, \ldots, n-r-1$, we obtain, $G^{\dagger} H=G^{\dagger}\left[\mathbf{u}_{1} \cdots \mathbf{u}_{n-r-1}\right]=\mathbf{0}$, which implies that $H^{T} \mathbf{w}_{0}=H^{T}\left(G^{\dagger}\right)^{T} \mathbf{c}=\left(G^{\dagger} H\right)^{T} \mathbf{c}=\mathbf{0}$. Thus, the expression of $\boldsymbol{\beta}$ appearing in (12) reduces to $\boldsymbol{\beta}=H^{T} B \mathbb{1}_{n}$.

Example 5 Let $A$ be the following reciprocal matrix

$$
A=\left[\begin{array}{cccc}
1 & 2 & 2 & 1 \\
1 / 2 & 1 & 3 & 1 \\
1 / 2 & 1 / 3 & 1 & 4 \\
1 & 1 & 1 / 4 & 1
\end{array}\right]
$$

We want to find the nearest consistent matrix to $A$, say $X=\left(x_{i j}\right)$, such that $x_{12}=x_{13}$ and $x_{13}=2 x_{24}$.

Let $Y=L(X)=\left(y_{i j}\right)$ be the solution to Problem 3. Since $x_{12}=x_{13}$ and $x_{13}=2 x_{24}$ one has $y_{12}-y_{13}=0$ and $y_{13}-y_{24}=\log 2$. These constraints lead to consider the following linear mappings $\Phi_{1}, \Phi_{2}: \mathscr{M}_{4,4} \rightarrow \mathbb{R}$ given by $\Phi_{1}\left(m_{i j}\right)=m_{12}-m_{13}$ and $\Phi_{2}\left(m_{i j}\right)=m_{13}-m_{24}$. To apply Theorem 6 , set $c_{1}=0$ and $c_{2}=\log 2$. The matrices $R_{1}, R_{2}$ such that $\Psi_{i}(M)=\left\langle M, R_{i}\right\rangle$ for all $M \in \mathscr{M}_{4,4}$ can be computed via Lemma 1:

$$
R_{1}=\left[\begin{array}{cccc}
0 & 1 & -1 & 0 \\
0 & 0 & 0 & 0 \\
0 & 0 & 0 & 0 \\
0 & 0 & 0 & 0
\end{array}\right], \quad R_{2}=\left[\begin{array}{cccc}
0 & 0 & 1 & 0 \\
0 & 0 & 0 & -1 \\
0 & 0 & 0 & 0 \\
0 & 0 & 0 & 0
\end{array}\right]
$$

The vectors $\mathbf{x}_{1}, \mathbf{x}_{2} \in \mathbb{R}^{4}$ and matrix $G \in \mathscr{M}_{4,2}$ can be easily found by using (9). The following computations can be easily performed by Octave, as is shown in the Appendix. Since $G^{T}\left(G^{T}\right)^{\dagger} \mathbf{c}=\mathbf{c}$, where $\mathbf{c}=\left[c_{1} c_{2}\right]^{T}$, by the criterion given in (11), the system $G^{T} \mathbf{w}=\mathbf{c}$ is consistent and a particular solution is $\mathbf{w}_{0}=\left(G^{T}\right)^{\dagger} \mathbf{c}$. Now, it is simple to finish this example by using Theorem 6 . Observe that in this example, $\boldsymbol{\beta}$ and $\boldsymbol{\mu}$ are scalars. The solution to Problem 3 is the matrix

$$
X=\left[\begin{array}{cccc}
1 & 2 & 2 & 2 \\
1 / 2 & 1 & 1 & 1 \\
1 / 2 & 1 & 1 & 1 \\
1 / 2 & 1 & 1 & 1
\end{array}\right]
$$

Obviously, this matrix satisfies $x_{12}=x_{13}$ and $x_{13}=2 x_{24}$.

Remark 3 If we have only one relationship to be fixed (i.e., in Theorem 6, one has $k=1$ ), an explicit expression for $\mathbf{w}_{0}$ (if it exists) can be given without using the Moore-Penrose inverse.

With the notation of Theorem 6 and under the assumption $\mathbf{x} \neq \mathbf{0}$ (otherwise, the set $\mathscr{A}$ defined in (7) is non empty if and only if $c=0$; if $c=0$, then $\mathscr{A}$ is the whole subset of $n \times n$ consistent matrices and Problem 3 was solved in [33, Theorem 2.3]), then $\left(G^{T}\right)^{\dagger}=\mathbf{x} /\|\mathbf{x}\|^{2}$. The consistency criterion given in (11) holds because

$$
G^{T}\left(G^{T}\right)^{\dagger}=\mathbf{x}^{T} \frac{\mathbf{x}}{\|\mathbf{x}\|^{2}}=1
$$

Hence we can choose the vector $\mathbf{w}_{0}$ appearing in Theorem 6 (see item c) of this same theorem) as

$$
\mathbf{w}_{0}=\frac{c}{\|\mathbf{x}\|^{2}} \mathbf{x} .
$$

Example 6 Let us see how Theorem 6 and the previous remark work with a rather artificial example. Let $A$ be the following reciprocal matrix:

$$
A=\left[\begin{array}{cccc}
1 & 2 & 4 & 1 \\
1 / 2 & 1 & 3 & 5 \\
1 / 4 & 1 / 3 & 1 & 4 \\
1 & 1 / 5 & 1 / 4 & 1
\end{array}\right]
$$


We want to find the consistent matrix nearest to $A$, say $X=\left(x_{i j}\right)$, such that $2 x_{12} x_{34}=x_{13}^{2}$.

To apply Theorem 6, we must consider $B=L(A)$. If the solution to Problem 3 is $Y=L(X)=\left(y_{i j}\right)$, since $2 x_{12} x_{34}=x_{13}^{2}$, then $y_{12}-2 y_{13}+y_{34}=-\log 2$, which leads to consider the linear mapping $\Psi: \mathscr{M}_{4,4} \rightarrow \mathbb{R}$ given by $\Psi(M)=m_{12}-2 m_{13}+m_{34}$. The matrix $R$ such that $\Psi(M)=\langle M, R\rangle$ for all $M \in \mathscr{M}_{4,4}$ can be computed by using Lemma 1:

$$
R=\left[\begin{array}{rrrr}
0 & 1 & -2 & 0 \\
0 & 0 & 0 & 0 \\
0 & 0 & 0 & 1 \\
0 & 0 & 0 & 0
\end{array}\right]
$$

The vector $\mathbf{x}$ defined in (9) is $\mathbf{x}=\left(R-R^{T}\right) \mathbb{1}_{4}=[-1,-1,3,-1]^{T} \neq \mathbf{0}$, and in this example, the matrix $G$ defined in (9) is $G=\mathbf{x}$. To apply Theorem 6 and Remark 3, we set $c=-\log 2$ and $\mathbf{w}_{0}=c \mathbf{x} /\|\mathbf{x}\|^{2}$. Therefore, the solution to Problem 3 is $Y=\phi_{4}\left(\mathbf{w}_{0}+H \boldsymbol{\mu}\right)$, where $H=\left[\mathbf{u}_{1} \mathbf{u}_{2}\right]$, the set $\left\{\mathbf{u}_{1}, \mathbf{u}_{2}, \mathbf{u}\right\}$ is an orthonormal basis of $\mathscr{N}\left(G^{T}\right), \mathbf{u}^{T} \mathbb{1}_{4} \neq 0$, and $\boldsymbol{\mu}$ is given in item $\mathrm{b}$ ) of Theorem 6 .

Finally, we compute the vector $\beta$ appearing in item a) of Theorem 6 by using item $c$ ), the vector $\mu$ given in (12), $Y=\phi_{4}\left(\mathbf{w}_{0}+H \mu\right)$, and $X=E(Y)$ is the solution given here.

$$
X=\left[\begin{array}{llll}
1.00000 & 1.01627 & 1.93355 & 3.55656 \\
0.98399 & 1.00000 & 1.90260 & 3.49964 \\
0.51718 & 0.52560 & 1.00000 & 1.83939 \\
0.28117 & 0.28574 & 0.54366 & 1.00000
\end{array}\right] .
$$

We can easily check that this matrix $X$ satisfies $2 x_{12} x_{34}=x_{13}^{2}$. To get a priority vector, it is enough to pick the normalised first row of $X$ or apply item $b$ ) of Theorem 6 . Further details can be found in the Appendix.

We research now a particular, but important, case: when just one entry (and the one on its symmetrical position) of a reciprocal matrix $A=\left(a_{i j}\right) \in \mathscr{M}_{n, n}$ is fixed. Evidently, we can assume $n>2$ (if $n=2$, the whole matrix would be fixed) and the fixed entries are $a_{12}$ and $a_{21}$. Recall that we use $\left\{\mathbf{e}_{1}, \ldots, \mathbf{e}_{n}\right\}$ for the standard basis of $\mathbb{R}^{n}$.

Theorem 7 Let $B \in \mathscr{M}_{n, n}$ be skew-symmetric and $n>2$. Let $\mathscr{A}=\left\{M=\left(m_{i j}\right) \in \mathscr{L}_{n}: m_{12}=b_{12}\right\}$.

a) $\mathscr{A} \neq \varnothing$ and $\mathscr{A}$ is a linear manifold whose dimension is $n-2$.

b) There exists a unique $Y \in \mathscr{A}$ such that $\|B-Y\|_{F}=\min \left\{\|B-Z\|_{F}: Z \in \mathscr{A}\right\}$ and is given by $Y=\phi_{n}\left(\mathbf{w}_{0}+\boldsymbol{\theta}\right)$, where

$$
\mathbf{w}_{0}=\frac{b_{12}}{2}\left(\mathbf{e}_{1}-\mathbf{e}_{2}\right), \quad \boldsymbol{\theta}=\frac{1}{2 n}\left[\begin{array}{cc}
0 & 0 \\
0 & 2 I_{n-2}+\mathbb{1}_{n-2} \mathbb{1}_{n-2}^{T}
\end{array}\right] B \mathbb{1}_{n} .
$$

Proof: To apply Theorem 3, let $R=\left(r_{i j}\right) \in \mathscr{M}_{n, n}$ be the matrix such that $r_{12}=1$ and $r_{i j}=0$ for $(i, j) \neq(1,2)$; and $c=b_{12}$. It is simple to see that the definition (9) yields $\mathbf{x}=\left(R-R^{T}\right) \mathbb{1}_{n}=\mathbf{e}_{1}-\mathbf{e}_{2} \neq \mathbf{0}$ and $G=\mathbf{x}$. By Theorem 3, we get item a). Also, we have by Remark 3

$$
\mathbf{w}_{0}=\frac{c}{\|\mathbf{x}\|^{2}} \mathbf{x}=\frac{b_{12}}{2}\left(\mathbf{e}_{1}-\mathbf{e}_{2}\right)
$$

The first assertion of item b) follows from Theorem 4. To apply Theorem 6 we need to find an orthonormal basis of $\mathscr{N}\left(G^{T}\right)$. It is evident that $\mathbf{v}=\left[v_{1} \cdots v_{n}\right]^{T} \in \mathscr{N}\left(G^{T}\right)=\left(\operatorname{span}\left\{\mathbf{e}_{1}-\mathbf{e}_{2}\right\}\right)^{\perp}$ if and only if $v_{1}=v_{2}$, i.e., we can choose $\left\{\mathbf{e}_{3}, \ldots, \mathbf{e}_{n},\left(\mathbf{e}_{1}+\mathbf{e}_{2}\right) / \sqrt{2}\right\}$ as an orthonormal basis of $\mathscr{N}\left(G^{T}\right)$. By employing the notation of Theorem 6 , we set $\mathbf{u}_{i}=\mathbf{e}_{i+2}$ for $i=1, \ldots, n-2, \mathbf{u}=\left(\mathbf{e}_{1}+\mathbf{e}_{2}\right) / \sqrt{2}$, and $H=\left[\mathbf{u}_{1} \cdots \mathbf{u}_{n-2}\right]$. Observe that $\mathbf{u}^{T} \mathbb{1}_{n} \neq 0$. 
It is simple to see that $H^{T} \mathbb{1}_{n}=\mathbb{1}_{n-2}$ and $\left\|H^{T} \mathbb{1}_{n}\right\|^{2}=\left\|\mathbb{1}_{n-2}\right\|^{2}=n-2$. Therefore, by items a) and c) of Theorem 6,

$$
\boldsymbol{\mu}=\frac{1}{n} \boldsymbol{\beta}+\frac{1}{n\left(n-\left\|H^{T} \mathbb{1}_{n}\right\|^{2}\right)} H^{T} \mathbb{1}_{n} \mathbb{1}_{n}^{T} H \boldsymbol{\beta}=\frac{1}{n} \boldsymbol{\beta}+\frac{1}{2 n} \mathbb{1}_{n-2} \mathbb{1}_{n-2}^{T} \boldsymbol{\beta}=\frac{1}{n} H^{T} B \mathbb{1}_{n}+\frac{1}{2 n} \mathbb{1}_{n-2} \mathbb{1}_{n-2}^{T} H^{T} B \mathbb{1}_{n} .
$$

Since $Y=\phi_{n}\left(\mathbf{w}_{0}+H \boldsymbol{\mu}\right)$, we will compute $H \boldsymbol{\mu}$. It is simple to see that

$$
H H^{T}=\left[\begin{array}{cc}
0 & 0 \\
0 & I_{n-2}
\end{array}\right], \quad H \mathbb{1}_{n-2}=\left[\begin{array}{c}
0 \\
\mathbb{1}_{n-2}
\end{array}\right], \quad \text { and } \quad H \mathbb{1}_{n-2} \mathbb{1}_{n-2}^{T} H^{T}=\left[\begin{array}{cc}
0 & 0 \\
0 & \mathbb{1}_{n-2} \mathbb{1}_{n-2}^{T}
\end{array}\right] \text {. }
$$

Hence

$$
\begin{aligned}
H \boldsymbol{\mu}=H\left(\frac{1}{n} H^{T} B \mathbb{1}_{n}+\frac{1}{2 n} \mathbb{1}_{n-2} \mathbb{1}_{n-2}^{T} H^{T} B \mathbb{1}_{n}\right) & =\frac{1}{n}\left[\begin{array}{cc}
0 & 0 \\
0 & I_{n-2}
\end{array}\right] B \mathbb{1}_{n}+\frac{1}{2 n}\left[\begin{array}{cc}
0 & 0 \\
0 & \mathbb{1}_{n-2} \mathbb{1}_{n-2}^{T}
\end{array}\right] B \mathbb{1}_{n} \\
& =\frac{1}{2 n}\left[\begin{array}{cc}
0 & 0 \\
0 & 2 I_{n-2}+\mathbb{1}_{n-2} \mathbb{1}_{n-2}^{T}
\end{array}\right] B \mathbb{1}_{n} . \square
\end{aligned}
$$

The function theorem7.m given in the Appendix can be used to find the solution to the problem solved in Theorem 7 (provided one has written the function phi.m given at the beginning of the Appendix).

\section{Conclusions}

Multi-criteria decision-making methods are used by individuals and enterprises to achieve effective solutions to many of their problems, since they usually include subjective, intangible, and not easily quantifiable aspects. Given the delicate features associated with DM in this context, the integration of as many tools as possible within MCDM methods to help the DM process is vital. In particular, a structured tool should consistently pave the way towards a good trade-off among diverse options. In this paper, we have considered the possibility of including expert hard-constraints in the feedback process for accommodating personal experience with the need to achieve acceptable consistency. We have specifically addressed this idea within the AHP framework.

Specifically, motivated by this fact - and tested by the authors in previous experience as facilitators in several DM processes - we considered the case in which an expert is unwilling to change one or more judgments when urged to do so by a synthetic consistency-enforcement method (such as the linearization method). Consequently, we solved the problem of achieving the closest consistent matrix to a given PCM, subject to the constraint of keeping one or more of the original entries unchanged. However, this practical and simple problem was extended to a more general context, namely that of imposing other constraints in conflict with some of the suggested modifications suggested by the consistency-enforcement tool. The classical Riesz representation theorem enables an ample generalization. Various lemmas and theorems give a solid and rigorous foundation for this approach. Using several examples, we have shown the effectiveness of the mechanism, which can be easily implemented in Octave. As a result, the developed tool can be directly applied to specific problems to achieve simultaneously, in a straightforward and effective way, consistency in a body of judgment within an AHP decision process and ad hoc adherence to expert opinion.

\section{Acknowledgments}

The use of English in the prose of this paper was revised by John Rawlins. 


\section{References}

[1] S. Safarzadeh, S. Khansefid, M. Rasti-Barzoki, A group multi-criteria decision-making based on best-worst method, Computers \& Industrial Engineering, 126 (2018) 111-121.

[2] A. Ishizaka, S. Siraj, Are multi-criteria decision-making tools useful? An experimental comparative study of three methods, European Journal of Operational Research, 264(2) (2018) 462-471.

[3] S. Carpitella, F. Carpitella, A. Certa, J. Benítez, J. Izquierdo, Managing human factors to reduce organisational risk in industry, Mathematical and Computational Applications, 23(4) (2018) 67.

[4] A. Gnanavelbabu, P. Arunagiri, Ranking of MUDA using AHP and Fuzzy AHP algorithm, Materials Today: Proceedings, 5 (5), Part 2 (2018) 13406-13412.

[5] X. Yu, S. Zhang, S, X. Liao, X. Qi, ELECTRE methods in prioritized MCDM environment, Information Sciences, 424 (2018) 301-316.

[6] A. Zareie, A. Sheikhahmadi, K. Khamforoosh, K, Influence maximization in social networks based on TOPSIS, Expert Systems with Applications, 108 (2018) 96-107.

[7] S. Carpitella, S.J. Ocaña-Levario, J. Benítez, A. Certa, J. Izquierdo, A hybrid multi-criteria approach to GPR image mining applied to water supply system maintenance, Journal of Applied Geophysics, 159 (2018) 754-764.

[8] K. Phudphad, B. Watanapa, W. Krathu, S. Funilkul, Rankings of the security factors of human resources information system (HRIS) influencing the open climate of work: using analytic hierarchy process (AHP), Procedia Computer Science, 111 (2017) 287-293.

[9] C. Bertolín, A. Loli, Sustainable interventions in historic buildings: A developing decision making tool, Journal of Cultural Heritage, 34 (2018) 291-302.

[10] R. Carli, M. Dotoli, R. Pellegrino, A decision-making tool for energy efficiency optimization of street lighting, Computers \& Operations Research, 96 (2018) 223-235.

[11] J. Huang, J. Boland, W. Liu, C. Xu, H. Zang, A decision-making tool for determination of storage capacity in grid-connected PV systems, Renewable Energy, 128, Part A (2018) 299-304.

[12] S.A. Erdogan, J. Šaparauskas, Z. Turskis, Decision Making in Construction Management: AHP and Expert Choice Approach, Procedia Engineering, 172 (2017) 270-276.

[13] L. Sheng, Y. Zhu, K. Wang, Uncertain dynamical system-based decision making with application to production-inventory problems, Applied Mathematical Modelling, 56 (2018) 275-288.

[14] R.R. Yager, Bidirectional possibilistic dominance in uncertain decision making, Knowledge-Based Systems, 133 (2017) 269-277.

[15] J. Benítez, S. Carpitella, A. Certa, J. Izquierdo, Characterisation of the consistent completion of AHP comparison matrices using graph theory, Journal of Multi-Criteria Decision Analysis, on line (2018) 1-13, DOI: 10.1002/mcda.1652.

[16] A. Kozierkiewicz-Hetmańska, The analysis of expert opinions' consensus quality, Information Fusion, 34 (2017) 80-86.

[17] I.E. Dror, J. Kukucka, S.M. Kassin, P.A. Zapf, P.A, When Expert Decision Making Goes Wrong: Consensus, Bias, the Role of Experts, and Accuracy, Journal of Applied Research in Memory and Cognition, 7(1) (2018) 162-163.

[18] V.S. Rekha, H. Muccini, Group decision-making in software architecture: A study on industrial practices, Information and Software Technology, 101 (2018) 51-63. 
[19] I. Montani, R. Marquis, N. Egli Anthonioz, C. Champod, Resolving differing expert opinions, Science \& Justice, In press (2018) https://doi.org/10.1016/j.scijus.2018.10.003.

[20] Z.P. Tian, R.X. Nie, J.Q. Wang, H.Y. Zhang, A two-fold feedback mechanism to support consensusreaching in social network group decision-making, Knowledge-Based Systems, In press (2018) https://doi.org/10.1016/j.knosys.2018.09.030.

[21] I.J. Pérez, F.J. Cabrerizo, S. Alonso, Y.C. Dong, F. Chiclana, E. Herrera-Viedma, On dynamic consensus processes in group decision making problems, Information Sciences, 459 (2018) 20-35.

[22] T.L. Saaty, The Analytic Hierarchy Process, McGraw-Hill, New York, 1980.

[23] Z.D. Unutmaz Durmuşoğlu, Assessment of techno-entrepreneurship projects by using Analytical Hierarchy Process (AHP). Technology in Society, 54 (2018) 41-46.

[24] S. Ozdemir, G. Sahin, Multi-criteria decision-making in the location selection for a solar PV power plant using AHP, Measurement, 129 (2018) 218-226.

[25] R.F.S.M. Russo, R. Camanho, Criteria in AHP: A Systematic Review of Literature, Procedia Computer Science, 55 (2015) 1123-1132.

[26] J. Franek, A. Kresta, Judgment Scales and Consistency Measure in AHP, Procedia Economics and Finance, 12 (2014) 164-173.

[27] T.L. Saaty, Decision-making with the AHP: Why is the principal eigenvector necessary, European Journal of Operational Research, 145 (2003) 85-91.

[28] T.L. Saaty, A scaling method for priorities in hierarchical structures, Journal of Mathematical Psychology, 15(3) (1977) 234-281.

[29] S. Bozóki, J. Fülöp, Efficient weight vectors from pairwise comparison matrices, European Journal of Operational Research, 264(2) (2018) 419-427.

[30] J. Szybowski, The improvement of data in pairwise comparison matrices, Procedia Computer Science, 126 (2018) 1006-1013.

[31] J. Benítez, S. Carpitella, A. Certa, A.E. Ilaya-Ayza, J. Izquierdo, Consistent clustering of entries in large pairwise comparison matrices, Journal of Computational and Applied Mathematics, 343 (2018) 98-112.

[32] J. Benítez, S. Carpitella, A. Certa, J. Izquierdo, C.M. La Fata, Some consistency issues in multicriteria decision making, Proceedings of the 22th Summer School "Francesco Turco", Palermo, Italy, September (2017) 13-15, 411-418.

[33] J. Benítez, X. Delgado-Galván, J. Izquierdo, R. Pérez-García, Achieving matrix consistency in AHP through linearization, Applied Mathematical Modelling, 35 (2011) 4449-4457.

[34] J. Benítez, X. Delgado-Galván, J.A. Gutiérrez-Pérez, J. Izquierdo, Balancing Consistency and Expert Judgment in AHP, Mathematical and Computer Modelling, 54 (2011) 1785-1790.

[35] T.L. Saaty, Fundamentals of decision making and priority theory with the analytic hierarchy process, Pittsburg: RWS Publication, 2000.

[36] L. Debnath, P. Mikusinski, Introduction to Hilbert spaces with applications, third ed., Academic Press, 2005.

[37] J. Benítez, X. Delgado-Galván, J. Izquierdo, R. Pérez-García, Improving consistency in AHP decision-making processes, Applied Mathematics and Computation 219 (2012) 2432-2441.

[38] J. Benítez, J. Izquierdo, R. Pérez-García, E. Ramos-Martínez, A simple formula to find the closest consistent matrix to a reciprocal matrix, Applied Mathematical Modelling 38 (2014) 3968-3974. 


\section{Appendix. Octave codes}

In this appendix we list useful Octave codes, that with minor changes, can be used to solve Problem 3 for concrete matrices. The first function computes $\phi_{n}(\mathbf{v})$ for $\mathbf{v} \in \mathbb{R}^{n}$ (recall that $\mathbf{v}$ is always a column) by using the definition (4).

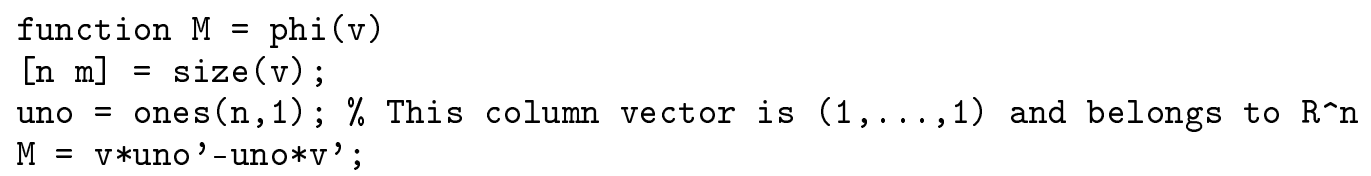

The following function was used to compute the solution to Problem 3 in Example 5.

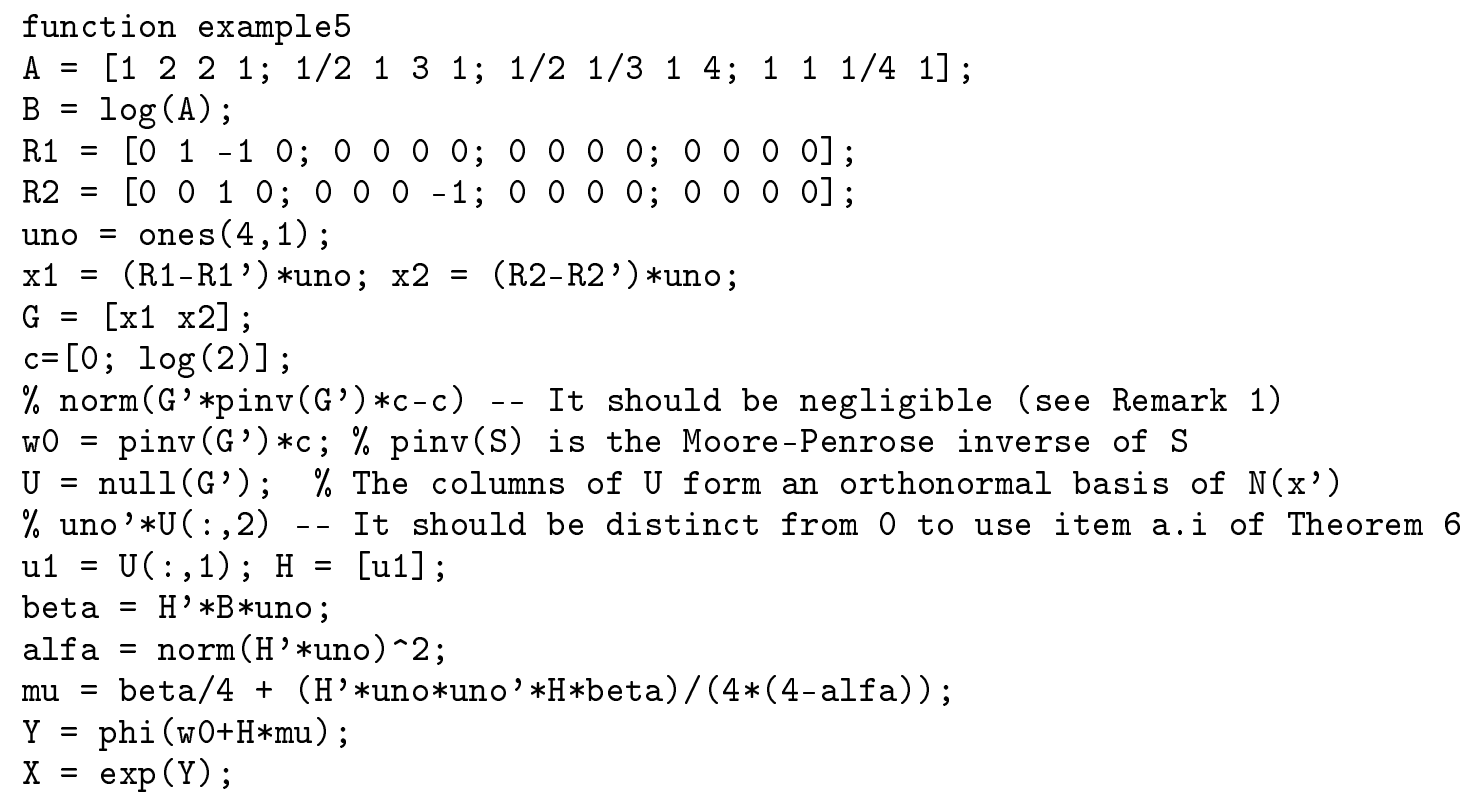

The following function was used in Example 6.

function example6

$A=\left[\begin{array}{lllllllllllllll}1 & 2 & 4 & 1 ; & 1 / 2 & 1 & 3 & 5 ; & 1 / 4 & 1 / 3 & 1 & 4 ; & 1 / 5 & 1 / 4 & 1\end{array}\right]$;

$B=\log (A)$;

$\mathrm{R}=\left[\begin{array}{cccccccccccccccc}0 & 1 & -2 & 0 ; & 0 & 0 & 0 & 0 ; & 0 & 0 & 0 & 1 ; & 0 & 0 & 0 & 0\end{array}\right]$;

uno $=\operatorname{ones}(4,1)$;

$\mathrm{x}=\left(\mathrm{R}-\mathrm{R}^{\prime}\right) *$ uno;

$\mathrm{C}=-\log (2)$;

$\mathrm{wO}=\mathrm{c} * \mathrm{x} / \operatorname{norm}(\mathrm{x}) \wedge 2$;

$\mathrm{U}=\operatorname{null}\left(\mathrm{x}^{\prime}\right) ; \%$ The columns of $\mathrm{U}$ form an orthonormal basis of $N\left(\mathrm{x}^{\prime}\right)$

$\%$ uno' $* U(:, 3)$ - - It should be distinct from 0 to use item a.i of Theorem 6 $\mathrm{u} 1=\mathrm{U}(:, 1) ; \mathrm{u} 2=\mathrm{U}(:, 2) ; \mathrm{H}=[\mathrm{u} 1 \mathrm{u} 2]$;

beta $=\mathrm{H}^{\prime} * \mathrm{~B} *$ uno;

alfa $=\operatorname{norm}\left(\mathrm{H}^{\prime} *\right.$ uno $) \wedge 2$;

$\mathrm{mu}=$ beta $/ 4+\left(\mathrm{H}^{\prime} *\right.$ uno $*$ uno' $* \mathrm{H} *$ beta $) /(4 *(4-\mathrm{alfa}))$;

$\mathrm{Y}=\operatorname{phi}(\mathrm{wO}+\mathrm{H} * \mathrm{mu})$;

$\mathrm{X}=\exp (\mathrm{Y})$

$\% 2 * X(1,2) * X(3,4) / X(1,3) \sim 2$ - - It should be 1 for this example.

The following function implements Theorem 7. 
function $X=$ theorem7 (A)

$B=\log (A)$;

$\left[\begin{array}{ll}\mathrm{n} & \mathrm{m}\end{array}\right]=\operatorname{size}(\mathrm{A})$;

w0 $=\mathrm{B}(1,2) *[1 ;-1 ; \operatorname{zeros}(\mathrm{n}-2,1)] / 2$;

$\operatorname{aux}=2 *$ eye $(n-2)+$ ones $(n-2, n-2)$;

theta $=[\operatorname{zeros}(2,2) \quad \operatorname{zeros}(2, \mathrm{n}-2) ; \operatorname{zeros}(\mathrm{n}-2,2)$ aux $] * \mathrm{~B} *$ ones $(\mathrm{n}, 1) /(2 * \mathrm{n})$;

$\mathrm{Y}=\mathrm{phi}(\mathrm{w} 0+$ theta $)$;

$\mathrm{X}=\exp (\mathrm{Y})$; 